\title{
Quality of Life Outcomes in 599 Cancer and Non-Cancer Patients with Colostomies
}

\author{
Robert Krouse, M.D., ${ }^{*}$ Marcia Grant, R.N., D.N.Sc., F.A.A.N., ${ }^{\dagger} 1$ Betty Ferrell, R.N., Ph.D., F.A.A.N., $\dagger$ \\ Grace Dean, R.N., Ph.D., ${ }^{*}$ Rebecca Nelson, Ph.D., $\neq$ and David Chu, M.D.* \\ *General and Oncologic Department of Surgery, $\dagger$ Department of Nursing Research and Education, $\ddagger$ Department of Biostatistics, \\ City of Hope National Medical Center, Nursing Research and Education, Duarte, California
}

Submitted for publication February 3, 2006

Background. A colostomy is known to impact negatively on a patient's quality of life (QOL). Concerns include incontinence, rectal discharge, gas, difficulties in returning to work, decreased sexual activity, and travel and leisure challenges. Reports have described QOL outcomes in cancer patients with colostomies and inflammatory bowel syndrome with colostomies, but little has been written regarding a comparison of cancer and non-cancer populations. The purpose of this study was to describe QOL issues of colostomy patients and compare these issues in cancer and non-cancer participants.

Materials and methods. A QOL-ostomy questionnaire was mailed to 2455 California members of the United Ostomy Association.

Results. Of the 1457 respondents (59\%), 599 had a colostomy. Most were results from cancer (517/599), with colorectal cancer being the most common diagnosis. The most common benign diagnoses were inflammatory bowel disease and diverticulitis. Demographics were similar, except for more females in the non-cancer group (76\%), and increased length of time with colostomy from the cancer group (mean 135.9 versus 106.4 months, $P=0.03)$. Common QOL problems included sexual problems, gas, constipation, travel difficulties, and dissatisfaction with appearance. Overall, cancer patients had less difficulty adjusting to their colostomies.

Conclusions. Results confirmed the negative impact of a colostomy on QOL. While patients with cancer had a better overall QOL than those with benign processes, concerns were common to all colostomy patients. These results provide health care practitioners with information useful in discussing QOL concerns during pre-

\footnotetext{
${ }^{1}$ To whom correspondence and reprint requests should be addressed at Nursing Research and Education, City of Hope National Medical Center, 1500 East Duarte Road, Duarte, CA 91010. E-mail: mgrant@coh.org.
}

operation treatment decisions and post operative teaching and follow-up care. @ 2007 Elsevier Inc. All rights reserved.

Key Words: Quality of life; Colostomy; Cancer; Noncancer.

\section{INTRODUCTION}

Colostomies are known to severely impact a patient's life regardless of the underlying diagnosis. Cohen et al., notes that for patients with cancer "concern about a colostomy frequently supersedes all other considerations of the patient." [1] This has also been demonstrated for patients with benign disease [2-6]. Colostomies for patients with cancer occur most frequently in patients with colorectal cancers, which are the third most common cancers in men (after prostate and lung cancer), and in women (after breast and lung cancer) [7]. While new sphincter-saving procedures (SSP) are done with increasing frequency, abdominoperineal resection (APR) with colostomy still remains a primary surgical approach for patients with newly diagnosed rectal cancer and patients with recurrent disease or complications. Colostomies for patients with non-cancer disease occur primarily because of complications of inflammatory bowel disease and diverticulitis, with many other less frequent causes.

Problems and concerns of patients with colostomies include incontinence, rectal discharge, inability to control gas, difficulties and adjustments of returning to work, decreased sexual activity, and difficulties in activities of travel and leisure [8,9]. Reports have described QOL outcomes in cancer patients with colostomies [10-20] and inflammatory bowel syndrome with colostomies, [21, 22] but little has been written regarding a comparison of cancer and non-cancer populations.

QOL is increasingly being regarded as an important end point for cancer management [23]. QOL is viewed as a multidimensional concept encompassing percep- 
tions of both positive and negative aspects of physical, emotional, social, cognitive, and spiritual functioning as well as the discomfort and symptoms produced by disease or treatment [24-26]. This definition views the patient as the authority for QOL data with information being derived from patient responses to QOL measurements. An understanding of the pattern of QOL concerns for patients with colostomies can provide the basis for needed clinical interventions [9]. As long term survival increases, different QOL concerns may emerge, and provide the basis for follow up care and rehabilitation. Although it can be hypothesized that there would be no difference between cancer and noncancer patients, a comparison of QOL in these patients can identify possible differences in the needs for these two groups.

Current concerns in conducting QOL studies focus on a lack of appropriate methods and instruments. As a result, inconsistent QOL results have occurred. CamilleriBrennan and Steele analyzed a large number of papers on QOL in patients with rectal cancer and concluded that most of the studies suffered from small sample sizes, questionnaires with unproven psychometric projections, and retrospective design [27].

The present study addresses these issues in several ways. A large population of colostomy survivors was included, a reliable and valid instrument specific to patients with colostomies was used, and patients answered QOL questions in relation to their current status.

This study used a comparative description design to identify differences in QOL concerns between patients with and without cancer who have colostomies. Study aims were as follows: 1) to identify common QOL concerns of patients with colostomies, 2) to compare QOL concerns of patients with and without cancer who have colostomies, 3) to analyze differences adjusting for demographic characteristics and time since colostomy.

\section{MATERIALS AND METHODS}

The conceptual framework for this study adapted a fourdimensional QOL model developed by the authors for evaluation of QOL in patients with cancer [28]. The dimensions include physical, psychological, social, and spiritual well-being and are defined in Table 1 [29]. The model has been adapted for use in a number of previous studies focusing on a variety of cancer populations [30-32]. Building on the original work conducted a number of years ago by Grant and colleagues, [33, 34] the current project involved revisions of the original QOL-Colostomy Questionnaire as described previously [35]. Briefly, data obtained from in-depth interviews of patients with ostomies were analyzed by a multidisciplinary team, and the results were used to revise the questionnaire. The multidisciplinary team consisted of one enterostomal nurse, two general surgeons, one urologist, and four nurse researchers. Following initial formation of the questionnaire, it was sent to a panel of three additional enterostomal nurses and one additional nurse researcher. Psychometric analysis was conducted on results of questionnaires received following a mailing to a large population of cancer and non-cancer patients with intestinal stomas. Specifics of this analysis are reported elsewhere [35]. The protocol for the current project was reviewed and
TABLE 1

Quality of Life Dimension Definitions

\begin{tabular}{ll}
\hline \multicolumn{1}{c}{ Dimension } & \multicolumn{1}{c}{ Definition } \\
\hline Physical well-being & $\begin{array}{c}\text { Physical symptoms and functional } \\
\text { ability } \\
\text { Emotional components of the illness } \\
\text { including positive as well as } \\
\text { negative aspects } \\
\text { Role of the patient within the family } \\
\text { and society including occupation, } \\
\text { social well-being }\end{array}$ \\
Spiritual well-being & $\begin{array}{c}\text { Religious and personal relationships } \\
\text { concerns }\end{array}$ \\
\hline
\end{tabular}

Created from data from Grant [29].

approved by the Institutional Review Board with receipt of mailed surveys accepted as informed consent.

The City of Hope QOL-Ostomy Questionnaire (COHQOL-OQ) was distributed to all members of the California United Ostomy Association. A total of 2455 questionnaires were mailed with 1457 returned for a response rate of $59 \%$. The response rate was calculated omitting questionnaires returned because of incorrect addresses or questionnaires from respondents with an unknown type of ostomy or an unknown diagnosis. Respondents had various types of intestinal stomas, including colostomies, ileostomies, and urinary diversions. This report focuses on colostomy patients, and includes 599 patients with and without cancer. Other groups will be described in future reports. Respondents were selected who indicated that they had a colostomy, and identified a diagnosis that clearly indicated cancer or no cancer. Respondents with ambiguous descriptions like "tumor" were not included in the analysis.

The COHQOL-OQ was used to measure QOL as well as other demographic and personal factors. The entire questionnaire may be completely downloaded from a City of Hope Website: (http://prc.coh.org) under Research Instruments, QOL Instruments, Ostomy. The psychometric analysis of the revised questionnaire confirmed the four dimension conceptual model (Table 1), and provided evidence for acceptable reliability and validity with Cronbach's alphas of the subscales ranging from 0.77 to 0.90 and Cronbach's alpha of the total questionnaire at $r=0.95$. The questionnaire is composed of two sections. The first section contains 46 descriptive questions used to identify demographic, disease, treatment, ostomy specific, and other personal characteristics including diet, work, and activity. The second section is composed of 43 items, divided into the four dimensions. Items under each dimension are scaled from 0 to 10 , with 0 being the worst QOL, and 10 being the best QOL. Overall scores for each dimension are computed by summing items in each subscale and dividing by the number of items in that subscale. Likewise, a total QOL measure is computed by summing scores on all items and dividing by the total number of items. Coding for the comparative analysis is described below.

Statistical analyses were conducted using the JMP Statistical Discovery Software, version 4.0.0 final candidate 11, developed by the SAS Institute (SAS Institute Inc., Cary, NC). All $P$ values are two-sided, with $\alpha=0.05$. Univariate logistic regression methods were performed using the Pearson's Chi-square [2] statistic. Multivariate logistic regression methods were performed using the Wald's test statistic. Missing values were excluded from the analyses.

\section{RESULTS}

\section{Patient Demographic and Disease Characteristics}

All patients included indicated they had a permanent colostomy. Most patients had a colostomy because of can- 
cer (517 of 599) (Table 2), with colorectal cancer $(n=456$, $88 \%$ ) being the most common malignant diagnosis. For benign disease, the most common diagnoses were inflammatory bowel disease (IBD) $(n=33,40 \%)$ and diverticulitis/infection $(n=14,17 \%)$. Demographics of the two groups were similar, except that there were more females in the non-cancer group (76\% versus $51 \%, P \leq$ $0.0001)$ and cancer patients had their colostomies longer (mean 135.9 versus 106.4 months, $P=0.03$ ). Adjustments for gender and time since colostomy were made in the subsequent multivariate analyses to remove any potential confounding effects these factors may have on patients' QOL assessments.

\section{Patient Personal Characteristics and Behaviors}

Table 3 shows the number and percentage of colostomy patients with cancer and colostomy patients without can- cer who responded positively to questions related to sexual function, psychological support/concerns, clothing, and diet. No statistically significant differences were found for questions related to post-colostomy sexual function between the two groups. Both groups were less sexually active post-colostomy.

In terms of psychological support, the majority of patients in both groups belonged to an ostomy support group and talked with other ostomy patients. However, patients with cancer were less likely to belong to a non colostomy-specific support group $(P$ value $=0.05)$. Half of the non-cancer patients and almost half of the cancer patients reported being depressed after their colostomy. A small percentage (7\%) of cancer patients and a comparable percentage (10\%) of non-cancer patients reported having considered or attempted suicide. In general, colostomy patients with cancer had fewer problems

TABLE 2

Patient Demographic and Disease Characteristics

\begin{tabular}{|c|c|c|c|}
\hline & No. of cancer patients (\%) & No. of non-cancer patients (\%) & $P$ value \\
\hline$N$ & 517 & 82 & \\
\hline \multicolumn{4}{|l|}{ Sex } \\
\hline Male & $254(49)$ & $20(24)$ & \\
\hline Female & $263(51)$ & $62(76)$ & $<0.0001$ \\
\hline \multicolumn{4}{|l|}{ Age } \\
\hline Mean & 72.5 & 70.1 & N.S. \\
\hline SD & 10.4 & 15.0 & \\
\hline Range & 39-95 & $15-92$ & \\
\hline \multicolumn{4}{|l|}{ Time since colostomy } \\
\hline Mean (months) & 135.9 & 106.4 & 0.027 \\
\hline $\mathrm{SD}$ (months) & 110.4 & 84.6 & \\
\hline Range (years) & $<1-54$ & $<1-27$ & \\
\hline \multicolumn{4}{|l|}{ Race/Ethnicity } \\
\hline Caucasian & $472(91)$ & $78(95)$ & N.S. \\
\hline African-American & $1(<1)$ & $1(1)$ & \\
\hline Hispanic & $14(3)$ & $1(1)$ & \\
\hline Asian & $18(3)$ & $1(1)$ & \\
\hline Native American & $3(1)$ & $0(0)$ & \\
\hline Other & $3(1)$ & $0(0)$ & \\
\hline Missing & $6(1)$ & $1(1)$ & \\
\hline Malignant diagnoses $(N=517)$ & No. of patients $(\%)$ & & \\
\hline Colorectal cancer & $456(88)$ & & \\
\hline Anal cancer & $12(2)$ & & \\
\hline Gynecologic cancer & $9(2)$ & & \\
\hline Urologic cancer & $6(2)$ & & \\
\hline Other & $3(1)$ & & \\
\hline Cancer, not otherwise specified & $31(6)$ & & \\
\hline Benign diagnoses $(N=82)$ & & No. of patients $(\%)$ & \\
\hline Inflammatory bowel disease & & $33(40)$ & \\
\hline Diverticulitis/infection & & $14(17)$ & \\
\hline Neuromuscular disturbance & & $9(11)$ & \\
\hline Benign tumor & & $6(7)$ & \\
\hline Obstruction & & $5(6)$ & \\
\hline Ischemic & & $2(2)$ & \\
\hline Other $^{a}$ & & $13(16)$ & \\
\hline
\end{tabular}

\footnotetext{
${ }^{a}$ Other: congenital defect $(n=1)$, damaged bowel $(n=1)$, difficulty with bowel $(n=1)$, fistula $(n=1)$, gangrene $(n=2)$, intestinal malfunction $(n=1)$, perforated colon $(n=1)$, radiation enteritis $(n=1)$, rectal disturbance $(n=1)$, and ruptured colon $(n=3)$.
} 
TABLE 3

Comparisons of Personal Characteristics and Behaviors

\begin{tabular}{|c|c|c|c|}
\hline & Cancer (\% yes) & Non-cancer (\% yes) & Adjusted $P$ value ${ }^{a}$ \\
\hline \multicolumn{4}{|l|}{ Sexual function } \\
\hline Sexually active pre-colostomy & $360(73)$ & $46(58)$ & N.S. \\
\hline Sexually active post-colostomy (if sexually active pre-colostomy) & $207(58)$ & $29(63)$ & N.S. \\
\hline Sexually satisfied (if sexually active post-colostomy) & $147(71)$ & $19(66)$ & N.S. \\
\hline Problem keeping erection (if male and applicable) & $181(79)$ & $13(76)$ & N.S. \\
\hline \multicolumn{4}{|l|}{ Psychological support/concerns } \\
\hline Belong to colostomy support group & $382(76)$ & $62(77)$ & N.S. \\
\hline Talk to other colostomy patient & $347(69)$ & $47(59)$ & N.S. \\
\hline Depressed post-colostomy & $218(43)$ & $40(51)$ & N.S. \\
\hline Belong to another support group & $51(11)$ & $16(22)$ & 0.05 \\
\hline Considered/attempted suicide & $33(7)$ & $8(10)$ & N.S. \\
\hline \multicolumn{4}{|l|}{ Clothing } \\
\hline Changed clothing style & $240(48)$ & $50(63)$ & N.S. \\
\hline Location problem with colostomy & $147(30)$ & $30(38)$ & N.S. \\
\hline \multicolumn{4}{|l|}{ Diet } \\
\hline Adjust diet because of colostomy & $255(51)$ & $57(70)$ & 0.02 \\
\hline Change diet because of gas & $211(42)$ & $39(49)$ & N.S. \\
\hline Avoid carbonated beverages ${ }^{b}$ & $172(36)$ & $36(46)$ & N.S. \\
\hline Avoid dairy products ${ }^{b}$ & $61(12)$ & $17(22)$ & 0.04 \\
\hline Avoid eating snacks ${ }^{b}$ & $49(10)$ & $12(15)$ & N.S. \\
\hline Avoid eating vegetables ${ }^{b}$ & $43(9)$ & $14(17)$ & 0.02 \\
\hline Avoid eating fruits ${ }^{b}$ & $40(8)$ & $14(18)$ & 0.008 \\
\hline
\end{tabular}

${ }^{a}$ Adjusted for sex and time since colostomy.

${ }^{b}$ If applicable-some respondents did not have these items in their diet prior to their colostomy.

with clothing styles and the location of their colostomy, although these differences were not statistically significant. Diet-related issues were more problematic for noncancer patients, with a higher percentage of them reporting having made dietary adjustments $(P=0.02)$ and needing to avoid certain foods (dairy products: $P=0.04$; vegetables: $P=0.02$; and fruits: $P=0.008$ ).

\section{QOL Scales: Moderate to Severe QOL Impact}

Further analysis was done to detect differences between the cancer and non-cancer groups based on severity of impact on QOL. For this comparative analysis, QOL scores were summarized as follows: 0-3= severe impact on QOL, 4-6 = moderate impact on QOL, with mild problems of 7 and above being omitted. This follows the schema used by Serlin et al., in the analysis of pain scores [36].

Table 4 shows the percentage of patients who reported having moderate to severe QOL concerns (defined as a rating of lower than seven on a 10-point scale, where ten is highest QOL). Items are organized within the four QOL dimensions and ranked for cancer patients from the greatest to the least percentage of responses indicating moderate to severe QOL impact. These questions reflect the four scales of physical, psychological, social/sexual, and spiritual dimensions of QOL. In general, it was found that colostomy patients with cancer reported having an overall higher QOL than patients with benign diagnoses. This was shown in the overall QOL scores, with $48 \%$ of non-cancer patients having scores that reflect a moderate to severe QOL impact as compared to only $32 \%$ of the cancer patients $(P=0.01)$.

The overall physical QOL subscale was significantly better for cancer patients, with only $22 \%$ of these subjects having a low physical QOL score as compared to $43 \%$ of the benign patients $(P=0.0008)$. For specific items, patients with a benign diagnosis reported having more problems by multivariate analysis with fatigue $(P=0.0001)$, strength $(P \leq 0.0001)$, sleep $(P=$ $0.02)$, overall physical well-being $(P=0.02)$, skin surrounding their colostomy $(P=0.007)$ and aches or pains $(P=0.005)$.

The overall psychological subscale revealed more non-cancer patients having a low psychological QOL score (50\% versus $37 \%$; adjusted $P=0.0001$ ). The following items were statistically lower in non-cancer patients even after adjustment for gender and time since colostomy: satisfaction with appearance $(P=0.05)$, feelings of usefulness $(P=0.02)$, anxiety $(P=0.004)$, depression $(P=0.05)$, and difficulty caring for colostomy $(P=0.01)$.

The overall social subscale showed that $41 \%$ of patients without cancer as opposed to $25 \%$ of those with cancer had a low social/sexual QOL score $(P=0.005)$. Multivariate analysis showed QOL scores in the areas of social functioning $(P=0.03)$, personal relationships $(P=0.01)$, interpersonal support $(P=0.01)$ and finan- 


\section{TABLE 4}

Comparison of Cancer and Non-Cancer Respondents with Moderate-to-Severe QOL Scores $(<7$ on a 10-point scale)

Cancer $\%$ Non-cancer $\%$ Adjusted patients $<7 \quad$ patients $<7 \quad P$ value $^{a}$

\begin{tabular}{|c|c|c|c|}
\hline \multicolumn{4}{|l|}{ Physical } \\
\hline Gas & 40 & 38 & N.S. \\
\hline Odor & 32 & 39 & N.S. \\
\hline Fatigue & 27 & 50 & 0.0001 \\
\hline Diarrhea & 26 & 33 & N.S. \\
\hline Strength & 25 & 50 & $<0.0001$ \\
\hline Sleep disruptions & 24 & 38 & 0.02 \\
\hline Overall well-being & 23 & 38 & 0.02 \\
\hline Leaking & 21 & 30 & N.S. \\
\hline Skin around colostomy & 20 & 35 & 0.007 \\
\hline Aches or pains & 18 & 34 & 0.005 \\
\hline Constipation & 14 & 11 & N.S. \\
\hline SUBSCALE & 22 & 43 & 0.0008 \\
\hline \multicolumn{4}{|l|}{ Psychological } \\
\hline \multicolumn{4}{|l|}{ Satisfaction with } \\
\hline Memory & 40 & 48 & N.S. \\
\hline \multicolumn{4}{|l|}{ Fearful of disease } \\
\hline recurring & 32 & 27 & N.S. \\
\hline Feel useful & 32 & 46 & 0.02 \\
\hline Feelings of control & 32 & 40 & N.S. \\
\hline Anxiety & 31 & 50 & 0.004 \\
\hline \multicolumn{4}{|l|}{ Difficulty adjusting to } \\
\hline \multicolumn{4}{|l|}{ Satisfaction/enjoyment in } \\
\hline life & 29 & 36 & N.S. \\
\hline Overall QOL & 28 & 36 & N.S. \\
\hline Embarrassment & 27 & 36 & N.S. \\
\hline Depression & 23 & 34 & 0.05 \\
\hline \multicolumn{4}{|l|}{ Difficulty caring for } \\
\hline colostomy & 17 & 30 & 0.01 \\
\hline Difficulty meeting people & 15 & 23 & N.S. \\
\hline \multicolumn{4}{|l|}{ Difficulty looking at } \\
\hline colostomy & 13 & 16 & N.S. \\
\hline SUBSCALE & 37 & 50 & 0.04 \\
\hline \multicolumn{4}{|l|}{ Social } \\
\hline Intimacy & 45 & 51 & N.S. \\
\hline Recreation/sports & 41 & 52 & N.S. \\
\hline Ability to travel & 37 & 49 & N.S. \\
\hline Privacy when traveling & 36 & 45 & N.S. \\
\hline Family distress & 33 & 41 & N.S. \\
\hline Social & 27 & 38 & 0.03 \\
\hline Personal relationships & 22 & 35 & 0.01 \\
\hline Isolation & 21 & 27 & N.S. \\
\hline Support & 21 & 33 & 0.01 \\
\hline \multicolumn{4}{|l|}{ Financial burden from } \\
\hline colostomy & 21 & 36 & 0.01 \\
\hline Privacy at home & 10 & 8 & N.S. \\
\hline SUBSCALE & 25 & 41 & 0.005 \\
\hline \multicolumn{4}{|l|}{ Spiritual } \\
\hline Positive changes & 58 & 37 & 0.002 \\
\hline Religious support & 39 & 39 & N.S. \\
\hline Uncertainty about future & 39 & 44 & N.S. \\
\hline Feelings of hope & 28 & 34 & N.S. \\
\hline Spirituality & 27 & 32 & N.S. \\
\hline Inner peace & 26 & 32 & N.S. \\
\hline Reason for living & 24 & 26 & N.S. \\
\hline SUBSCALE & 38 & 43 & N.S. \\
\hline TOTAL QOL & 32 & 48 & 0.01 \\
\hline
\end{tabular}

${ }^{a}$ Adjusted for sex and time since colostomy. cial burden from colostomy $(P=0.01)$ were all statistically significantly lower for patients with a benign diagnosis.

Of all of the QOL dimensions, assessment of spiritual QOL was the most similar between cancer and non-cancer patients with no statistically significant difference in the spiritual QOL subscale. The question, "Has having an colostomy made positive changes in your life?" was the only item in this dimension that differed significantly across groups, with fewer cancer patients reporting a lower score (less positive changes) than non-cancer patients $(P=0.002)$. This is the only item in which cancer patients had a poorer QOL than non-cancer patients.

\section{DISCUSSION}

Although the effectiveness of medical treatment has traditionally been determined by endpoints such as long-term, overall, or relapse-free survival as well as response rate, time to treatment failure, and time to progression, QOL is increasingly being regarded as an important end point especially for cancer management [23]. Previous studies on QOL in patients with colostomies have primarily reported on issues in cancer patients or issues in non-cancer patients. In contrast, we compared QOL results across cancer and noncancer patients with cancer patients averaging 10 years experience with an ostomy and non-cancer patients averaging more than 8 years experience. Despite this long time for adjustment, both cancer and noncancer patients reported persistent problems across all QOL dimensions.

Most QOL studies for stoma patients suffer from small samples with subjects ranging from 21 to 260 [13-20]. The rate of questionnaire return in our report was quite high (1457/2455, 59\%), adding to the generalizability of the results. It must be acknowledged that there will be a selection bias in our study based on the surveyed population, as well as the fact that respondents are likely a highly motivated group. Also, because over $95 \%$ of our respondents were Caucasian, the utility of these results to other racial groups may be somewhat limited. In addition, because our study used a mailing list from the United Ostomy Association California Division, we do not have information available on non-responders. Neither did we have support for this study to use a control group of either nonpatients, or patients undergoing major abdominal surgery without a colostomy. These approaches are being used in our current funded studies. In addition, in this anonymous, mailed survey we did not have access to medical record information.

This study did not include a survey of norms for a non-patient population. Historically, we did use our general survey in 165 employees from the City of Hope National Medical Center (consisting of the four sub- 
scales but no ostomy-specific items). While the overall HR-QOL is lower for all patients with ostomies no matter whether they were cancer or non-cancer patients, one must be very cautious in comparing results to non-patients as the survey is slightly different, and the populations may not be comparable.

Patients in the cancer and non-cancer groups had similar characteristics, except for the predominance of females in the benign group. This may be related to the fact that the inflammatory bowel diseases affect females more often than males. For patients with a history of colorectal cancer, there were similar numbers of men (229) and women (227), which mirrors the colorectal cancers nationally for men $(71,820)$ and women $(73$, 470) [7]. In addition the length of time with colostomy was greater for the cancer group. Gender and time with colostomy were thus in our subsequent stratified analyses. The number of cancer patients far exceeded the number of non-cancer patients. This is not surprising in that patients with ulcerative colitis, especially before sphincter-saving procedures becoming a standard of care, usually received an ileostomy rather than a colostomy.

The overall QOL was significantly better for cancer patients when compared with non-cancer patients when adjusted for gender and time since colostomy. This is also seen in the physical, psychological and social subscales, although no difference was noted for the spiritual subscale. These differences may reflect to differences in general health. Given the time since surgery, the cancer patients were likely cured of their original disease whereas those with non-cancer diagnoses, such as IBD may have continued disease exacerbations and remissions [2-6]. The non-cancer patients may also have had worse pre-stoma QOL with multiple GI symptoms, probably accounting for less sexual activity pre-colostomy ( $73 \%$ versus $58 \%)$.

A large majority of both cancer $(76 \%)$ and non-cancer (77\%) respondents belong to ostomy support groups. In addition, cancer patients tended to talk to other colostomy patients more (69\% versus $59 \%$ ), and non-cancer patients tended to belong to other support groups. In our qualitative analysis of patient interviews, [35] a common theme indicated that patients found group and individual discussion to be helpful. We have seen with bone marrow treatment survivors [37] and breast cancer treatment survivors [38] the need for counseling, discussion, and information.

Many general QOL difficulties are either related directly or indirectly to a colostomy. Grant et al., assessed QOL in cancer patients with colostomies, and found that patients with colostomies had the lowest QOL scores when compared to other outpatients receiving either chemotherapy or radiation therapy [33]. Only inpatients receiving chemotherapy had poorer QOL scores than the patients with colostomies. That study established fatigue, work, physical strength, sexual activity, and adjustment as some of the areas of most concern for patients with colostomies.

QOL evaluation relating to the physical domain revealed common colostomy-specific concerns of gas, odor, diarrhea, and leakage around the stoma or appliance. These results are similar to those found in previous studies and may lead to psychological and social concerns $[8,39,40]$. Multiple differences in the physical domain occur between cancer and non-cancer patients. First, non-cancer patients reported lower scores for general QOL items such as fatigue, strength, and aches and pains, which may be more reflective of ongoing medical problems rather than the colostomy itself. The higher incidence of sleep difficulties in the non-cancer group may actually be related to the colostomy, as qualitative interviews, confirmed that sleep problems were related to the inability to sleep on the abdomen and a realistic fear of leakage from the applicance [9, 35] Skin irritation occurred more frequently in the benign disease group. Although not statistically significant, diarrhea and leakage from the appliance were reported more frequently in the benign group. These results may be related to ongoing disease in the non-cancer group.

Although a high percentage in each group altered their diet, this was more of an issue for non-cancer patients. This difference may be associated with dietary changes needed for patients with IBD or diverticulitis. More non-cancer patients avoid certain foods, including dairy products, fruits, and vegetables. In addition, both groups reported needing to change clothing styles and having location problems with their stomas. Clothing changes may be associated with location of the stoma, body habitus, weight changes, and the kind of appliance used. Problem with the location of the colostomy could be associated with poor placement, development of a hernia, leakage from poor appliance application, and changes in the patient's body contour over time. These are important factors to recognize during patient teaching pre- and post-operatively, as well as during long term follow-up.

Multiple problems of colostomy patients are found in the psychological domains related to the stoma, although the severity is often worse in the non-cancer category. Satisfaction with appearance is the most common QOL dilemma for both groups, although significantly worse with non-cancer respondents. Of note, neither group reported problems looking at their own stoma. This may be related to a combination of time since surgery and experience with daily care. Respondents still can have persistent dissatisfaction with their general appearance or body image. Orbach and Tallent found that poor body image persisted in colostomy patients 5 to 10 years after their surgery [41]. Patients with cancer have reported lower scores for body image before their 
colostomy compared to 6 months after surgery, showing the negative psychological effects a stoma can have even before the operation [16]. In that study, the authors suggest that this may have to do with both the colostomy as well as the diagnosis of cancer.

In our study, depression and anxiety were significantly higher in the non-cancer group. Forty-three percent in the cancer group and 51\% in the non-cancer group affirmed that they had depression after placement of their colostomy, although less regarded their depression as moderate or severe. Patients in either group rarely considered suicide ( $7 \%$ cancer, $10 \%$ noncancer), although this could be considered clinically significant. In a comparison study of cancer patients, it has been shown that colostomy patients had more depression and hopelessness both pre- and postoperatively when compared with patients who had their bowel reasnastamosed [10]. Although the diagnosis of cancer did impact both groups, a colostomy had a greater effect on emotional state.

Fear of recurrence in the cancer group was a moderate or severe problem for $32 \%$ and not significantly different from the non-cancer group. This issue was not further probed in the survey, because most patients in each group were far removed from their original procedures. For cancer patients, those who did not have a recurrence of their cancer found that survival alone made coping with the colostomy easier [40]. For chronic diseases such as Crohn's disease, continued concerns related to their disease is a well known issue for these patients, [2] and is a contributing factor to well known psychological disturbances in this population [42].

For social well-being, there are several common problems that impinge on QOL for both cancer and noncancer groups. Recreation activities, including playing sports and traveling, are among the most problematic issues for all respondents. Intimacy is the most severe social issue for colostomy patients. Family distress is a dilemma for many respondents, and is somewhat worse in the non-cancer group. Non-cancer patients have significantly more problems with personal relationships and a social support system. It is unclear why non-cancer patients feel that colostomies are more of a financial burden, but may be associated with long-term health insurance coverage. In any case, financial burden is clearly a source of concern for many colostomy patients.

In our study, approximately one-half of both cancer and non-cancer patients did not have sexual relations after their ostomy was placed. The reasons may be multifactorial, including the physical problems related to leakage, odor, or gas and feces filling the colostomy bag. Psychological issues, such as satisfaction with appearance and embarrassment undoubtedly are a component of sexual and intimacy problems. Nerve damage related to the surgery itself will play roles with both male and female sexual abilities and satisfaction. In a review of the literature, Weber and Petrelli found that APR for rectal cancer is associated with retrograde ejaculation in 3 to $39 \%$, and impotence in 15 to $100 \%$ [43]. Erectile dysfunction was high in our study, with no difference between cancer patients $(79 \%)$ and non-cancer patients (76\%). Sexual dysfunction for women after colorectal surgery needs further study [44].

To improve QOL and keep fecal flow normal, surgeons are now using a low anastomosis where possible instead of APR for both malignant and benign disorders, although it is not conclusive that patients actually have an improved QOL. Grumann et al., actually report that QOL is consistently more favorable for patients who underwent an APR versus a LAR [45]. In fact, they showed APR patients had significantly better scores for variables related to sleep disturbances, diarrhea, and constipation. In comparison, a study of patients with APR and SSP for low rectal cancers found that SSP patients returned to work sooner, and reported fewer problems with flatus, odor, dietary restrictions, and depression [14]. There were no reported differences in anxiety or "problems with vacation activities," although colostomy patients had more difficulties with "leisure pursuits." In addition, no difference was found in male sexual function between groups $(P=0.06)$ [14]. Stoma patients tended to have more difficulties with erections and fewer were sexually active after surgery. In a review of 17 studies conducted from 1987 to 1992 comparing stoma versus non-stoma patients with cancer, Sprangers et al., confirmed these findings [8].

Data from this study and the literature provide the basis for teaching and counseling patients with stomas. These include interventions to decrease gas, odor, and loose bowel movements, and help with peristomal skin care [46]. In addition, sexual function can be enhanced through many suggestions, emotional support, or urologic interventions [47]. The suggestion by Dukes in 1947 for counseling of new or future patient by longterm ostomates is still an important component of care [48]. Watson studied the impact of a short-term post operative counseling on cancer patients with ostomies. Thirty-one ostomy patients were randomized to receive either short-term counseling or the usual care. Evaluations at 1 month post-surgery revealed that patients who received counseling were more independent, more involved in activities outside the home and had improved self feelings when compared with the control group [20]. Although studies may differ as to the impact of age of psychological function, [8, 49] it has been shown that older patients may display different coping styles compared to younger patients. Therefore, methods of intervention should be geared toward a patient's age [49]. Financial aid should be addressed pre- 
operatively to avoid undue anxieties, especially for patients with benign diseases.

In conclusion, surgeons can anticipate multiple problems for both cancer and non-cancer patients before and after a colostomy procedure. Appropriate surgical placement of colostomies and pre and post-operative education have long-term effects on patients and their families. Understanding what other issues persist for these patients can ensure they are considered and addressed in follow-up. In fact, when patient education regarding the colostomy is begun in the pre-operative period, patients may require less time and have fewer problems in their rehabilitation [20]. Thus, access to informed surgeons and ostomy/wound care nurses may prevent and/or alleviate many of these short and longterm problems. Testing such resources in future studies is an appropriate next step. This educational preparation is the responsibility of the surgeon, with support from the enterostomal therapist and the clinical care team [46]. Interventions regarding rehabilitation should continue soon after surgery and colostomy treatments should be individualized based on a patient's underlying disease state. Finally, we must also consider the possibility that in some instances, a colostomy can lead to an improved HR-QL if issues are addressed early through post-operative support and education.

\section{ACKNOWLEDGMENTS}

We thank the assistance of several ostomy and wound nurses who participated in the development of the questionnaire used in this study: Doreen Bergstrom, R.N., E.T., City of Hope National Medical Center; Betty Razor, R.N., E.T., Private Practice; Margaret Landry, R.N., CWOCN, Private Practice; and Barbara Bates-Jensen, R.N., Ph.D., CETN, University of California Los Angeles.

This research was supported by a grant from the NIH/NCI. CA 72666: Quality of Life for Cancer Patients with Ostomies and the City of Hope Cancer Center Support Grant CA 33572.

\section{REFERENCES}

1. Cohen AM, Minsky BD, Schilsky RL. Cancer of the colon In: Devita LT, Hellman S, Rosenbeng SA, eds. Cancer: Principles and practice of oncology, 5th ed. Philadelphia: Lippincolt, 1997; 1163.

2. Yazdampanah Y, Klein O, Gambiez L, et al. Impact of surgery on quality of life in Crohn's disease. AMJ Gastroent 1997;92: 1897.

3. Moser G, Tillnger W, Sachs G, et al. Disease-related worries and concerns: A study on out-patients with inflammatory bowel disease. Eur J Gastroenterol Hepatol 1995;7:853.

4. Drossman DA, Leserman J, Li Z, Mitchell CM, Zagumi EA, Patrick DL. The rating form of IBD patient concerns: A new measure of health status. Psycosom Med 1991;53:701.

5. Irvine EJ. Quality of life issues in patients with inflammatory bowel disease. Am J Gastroenterol 1997;92:18S.

6. Hjortswang H, Strom M, Almer S. Health-related quality of life in Swedish patients with ulcerative colitis. Am J Gastroenterol 1998;93:2203.
7. Cancer Facts and Figures 2005. American Cancer Society. CA: A Cancer Journal for Clinicians. 2005;55:10.

8. Sprangers MAG, Taal BG, Aaronson NK, te Velde A. Quality of life in colorectal cancer: Stoma vs. non stoma patients. Dis Colon Rectum 1995;38:361.

9. Grant M. Quality of life issues in colorectal cancer. Develp Support Care 1999;3:4.

10. Wirsching M, Druner HU, Herrmann G. Results of psychosocial adjustment to long- term colostomy. Psychother Psychosom 1975;26:245.

11. MacDonald LD, Anderson HR. Stigma in patients with rectal cancer: A community study. J Epidemiol Community Health 1984;34:284.

12. Hollander J, Gonnella C, Parker D. Functional recovery from cancer surgery: Estimation of expectations. Arch Phys Med Rehabil 1979;60:45.

13. Koukouras D, Spiliotis J, Scopa CD, Kalfarentzos F, Tzoracoleftherakis E, Androulakis J. Radical consequence in the sexuality of male patients operated for colorectal carcinoma. Eur J Surg Oncol 1991;17:285.

14. Williams NS, Johnston D. The quality of life after rectal excision for low rectal cancer. Br J Surg 1983;70:460.

15. LaMonica G, Audisio RA, Tamburini M. Incidence of sexual dysfunction in male patients treated surgically for rectal malignancy. Dis Colon Rectum 1985;28:937.

16. Jenks JM, Morin KH, Tomaselli N. The influence of ostomy surgery on body image in patients with cancer. Appl Nurs Res 1997;10:174.

17. Fazio VW, Fletcher J, Montague D. Prospective study of the effect of resection of the rectum on male sexual function. World J Surg 1980;4:149.

18. Santangelo ML, Romano G, Sassaroli C. Sexual function after resection for rectal cancer. Am J Surg 1987;154:502.

19. Sutherland AM, Orbach CE, Dyk RB, Bard M. The psychological impact of cancer and cancer surgery: I. Adaption to the dry colostomy: Preliminary report and summary of findings. Cancer 1952;5:857.

20. Watson PG. The effects of short-term post-operative counseling on cancer/ostomy patients. Cancer Nursing 1983;3:21.

21. Lask B, Jenkins J, Nabarro L, Booth I. Psychosocial sequealae of stoma surgery for inflammatory bowel disease of childhood. Gut 1987;28:1257.

22. Stahlgren L, Ferguson K. Effects of abdominoperineal resection on sexual function in sixty patients with ulcerative colitis. Arch Surg 1959;78:604.

23. Decosse JJ. Quality of life. J Surg Oncol 1997;65:321.

24. Osoba D. Lessons learned from measuring health-related quality of life in oncology. J Clin Oncol 1994;12:609.

25. Grant MM, Padilla GV, Ferrell BR, Rhiner M. Assessment of quality of life with a single instrument. Semin Oncol Nurs 1990;6:260.

26. Spilker B. Introduction. In: Spilker B, ed. Quality of life and pharmacoeconomics in clinical trials, 2nd ed. Philadelphia: Lippincott-Raven, 1996;1-10.

27. Camilleri-Brennan J, Steele RJC. Quality of life after treatment for rectal cancer. Br J Surg 1998;85:1036.

28. Ferrell B, Grant M, Padilla G, Vermuri S, Rhiner M. The experience of pain and perceptions of quality of life: Validation of a conceptual model. Hospice J 1991;7:9.

29. Grant M. Assessment of QOL following hematopoietic cell transplantation. In: Forman SJ, Blum KG, Thomas ED, eds. Hematopoietic cell transplantation, 2nd ed. Oxford: Blackwell Science, Inc., 1999. pp. 407-413.

30. Grant MM, Ferrell B, Schmidt GM, Fonbuena P, Niland JC, 
Forman SJ. Measurement of quality of life in bone marrow transplantation survivors. Qual Life Res 1992;1:375.

31. Padilla G, Ferrell B, Grant M, Rhiner M. Defining the content domain of quality of life for cancer patients with pain. Cancer Nursing 1990;13:108.

32. Ferrell BR, Hassey-Dow K, Grant M. Measurement of the quality of life in cancer survivors. Qual Life Res 1995;4:523.

33. Grant MM, Padilla GV, Preasant C, Lipsett J, Runa PL. Cancer patients and quality of life. Proceedings of the Fourth National Conference on Cancer Nursing, 1984.

34. Padilla GV, Grant MM. Quality of life as a cancer nursing outcome variable. Adv Nurs Sci 1985;8:45.

35. Grant M, Ferrell B, Dean G, Uman G, Chu D, Krouse R. Revision and psychometric testing of the City of Hope Quality of Life-Ostomy questionnaire. Qual Life Res 2004;13:1445.

36. Serlin RC, Mendoza TR, Nakamura Y, Edwards KR, Cleeland CS. When is cancer pain mild, moderate or severe? Grading pain severity by its interference with function. Pain 1995;61: 277.

37. Ferrell B, Grant M, Schmidt GM, Rhiner M, Whitehead C, Fonbuena P, Foreman SJ. The meaning of quality of life for bone marrow transplant survivors Part 2. Improving quality of life for bone marrow transplant survivors. Cancer Nurs 1992; 15:247-253.

38. Ferrell BR, Grant MM, Funk B, Otis-Green S, Garcia N. Quality of life in breast cancer survivors as identified by focus groups. Psycho Oncol 1997;6:13.
39. Bartha I, Hadju J, Bokor L, Kanyuri Z, Damjanovice L. Quality of life of post-colostomy patients. Orvosi Hetilap 1995;136:1995.

40. Sahay TB, Gray RE, Fitch M. A qualitative study of patient perspectives on colorectal cancer. Cancer Practice 2000;8:38.

41. Orbach CE, Tallent N. Modification of perceived body and of body concepts following the construction of a colostomy. Arch Gen Psych 1965;12:126.

42. Schwartz RA, Schwartz IK. Psychiatric disorders associated with Crohn's disease. Int J Psych Med 1982;12:67.

43. Weber TK, Petrelli NJ. Local excision for rectal cancer: An uncertain future. Oncology 1998;12:933.

44. Ofman US, Auchincloss SS. Sexual dysfunction in cancer patients. Curr Opin Oncol 1992;4:605.

45. Grumann MM, Noak EM, Hoffmann IA, Schlag PM. Comparison of quality of life in patients undergoing abdominoperineal extirpation of anterior resection for rectal cancer. Editorial Ann Surg 2001;223:149.

46. Kurtzman SH, Gardner B, Kellner WS. Rehabilitation of the cancer patient. Am J Surg 1988;155:791.

47. Grunberg KJ. Sexual rehabilitation of the cancer patient undergoing ostomy surgery. J Enterostom Ther 1986;13:148.

48. Dukes CE. Management of a permanent colostomy: A study of 100 patients at home. Lancet 1947;2:12.

49. Keyes K, Bisno B, Richardson J, Marston A. Age differences in coping, behavioral dysfunction and depression following colostomy surgery. Gerontologist 1987;27:182. 